\title{
Journalismus studieren in der Krise
}

\author{
Thomas Hanitzsch / Corinna Lauerer / Nina Steindl
}

Eine deutschlandweite Befragung von 556 Journalismus-Studierenden hat untersucht, ob und inwiefern sich die aktuelle Krise im Journalismus in der hochschulgebundenen Journalistenausbildung niederschlägt. Die Ergebnisse zeigen, dass unter den Studierenden keine ausgeprägte Krisenstimmung herrscht, auch wenn eine Mebrbeit der Befragten die Zukunftschancen des Journalismus ambivalent bewertet. Die größten Herausforderungen für den Berufsstand werden in ökonomischen Faktoren sowie in verschlechterten Arbeitsbedingungen geseben. Selbstverwirklichung und Idealismus haben angesichts der schwierigen Bedingungen einen entscheidenden Einfluss auf die Studienfachwabl. Darüber hinaus zeigt sich, dass trotz schwieriger Zeiten immer noch eine klare Mebrbeit der Studierenden in den Journalismus möchte. Die Erfabrungen, die Studierende im redaktionellen Alltag machen, bestärkt sie dabei in ibrer Berufswabl und gibt ibnen zudem mebr Sicherheit hinsichtlich der Erreichbarkeit ibrer beruflichen Ziele. Gleichzeitig bereitet sich knapp die Hälfte der Studierenden im Geiste bereits darauf vor, in einem von beruflicher Mobilität gekennzeichneten Arbeitsmarkt irgendwann auch auf andere Tätigkeitsbereiche auszuweichen.

Schlüsselwörter: Journalistenausbildung, Hochschule, Journalismus-Studierende, Krise des Journalismus, diskursiver Institutionalismus

Aktuell kommt kaum ein Bericht zur Lage des deutschen Journalismus ohne Krisenrhetorik aus. Die Ursachen für die Krisen sind vielfältig: sinkende Auflagen und Marktanteile, die Abwanderung von Anzeigen in das Internet, zunehmende Konkurrenz durch kostenlose Online-Nachrichtenportale und Bürgerjournalismus sowie eine Veränderung des Nachrichtenkonsums insgesamt. Gerhardt, Kepplinger und Maurer haben im Feuilleton der FAZ deshalb bereits 2005 einen „Klimawandel in den Redaktionen“ konstatiert. Jede Journalismuskrise sei demnach auch eine Krise der demokratischen Öffentlichkeit.

Sowohl im politischen als auch im medialen sowie kommunikationswissenschaftlichen Diskurs ist das Krisennarrativ daher omnipräsent. So weist die EU-Charta „Normensetzung für die Freiheit der Medien in der EU“ in aller Deutlichkeit darauf hin, „dass eine steigende Zahl von Journalisten unter prekären Bedingungen beschäftigt wird und es ihnen an den sozialen Sicherheiten mangelt, die auf dem normalen Arbeitsmarkt üblich sind“ (Europäisches Parlament 2013). Frank Patalong (2015) beschrieb den journalistischen Arbeitsmarkt unlängst als „Krisestan“, während in der Journalismusforschung bereits über das „Ende“ sowie das „Sterben“ des Journalismus spekuliert wird (Charles \& Stewart 2011; McChesney \& Pickard 2010). Eine im Jahr 2008 an der University of Bedfordshire organisierte Konferenz widmete sich gar in voller Gänze dem Thema „The End of Journalism?" Da die allgemeine Verunsicherung unter Journalisten an allen Ecken und Enden zu spüren ist, drängt sich manchem die Frage auf, was Journalismus-Studierende im Angesicht der krisenhaften Entwicklungen motiviert, diesen Ausbildungsweg dennoch einzuschlagen.

Die hochschulgebundene Journalistenausbildung kann in Deutschland auf eine fast fünfzigjährige Tradition zurückblicken (Fröhlich \& Holtz-Bacha 2009). Anders als in den USA hat sich der Bedeutungsgewinn von Journalistik-Studiengängen seit den 1970er Jahren aber nur mäßig im Berufsstand manifestiert. Eine umfassende Journalistenbefra- 
gung aus dem Jahr 2005 zeigt, dass die Zahl der Journalisten mit einem JournalistikAbschluss bei ca. 14 Prozent liegt (Weischenberg, Malik \& Scholl 2006). Aktuelle Studierendenzahlen sind schwer zu erheben. Nach Angaben des Bundesamts für Statistik waren in den kommunikations- und publizistikwissenschaftlichen Fächern im Wintersemester 2015/16 deutschlandweit knapp über 17.500 Studierende eingeschrieben. Fest steht, dass sich die Ausbildungslandschaft u. a. im Zuge der Bologna-Umstellung relativ breit aufgefächert und thematisch ausdifferenziert hat (Harnischmacher 2010a). Führt man die Daten aus dem Hochschulkompass und Medienstudienführer zusammen ${ }^{1}$, finden sich aktuell 25 grundständige und 24 Master-Studiengänge an deutschlandweit 32 Hochschulen, die den Begriff "Journalismus" oder "Journalistik“ in der Studiengangsbezeichnung führen.

Anhand einer deutschlandweiten Befragung von 556 Studierenden, die 2014 in einschlägigen Journalistik-Studiengängen eingeschrieben waren, geht dieser Beitrag der Frage nach, wie deutsche Studierende die Zukunftschancen des Journalismus bewerten, wo sie die größten Probleme sehen, was sie motiviert, Journalist zu werden, in welchem Medienbereich sie arbeiten möchten, inwiefern sie ihre Karriere im Journalismus pessimistisch sehen und ob sich diese Einschätzungen und Karriereziele im Laufe des Studiums verändern.

\section{Journalismus als Diskurs steht unter Druck}

Betrachtet man Journalismus als Institution, deren Identität und Funktion gesellschaftlich verhandelt wird, stellt sich die Frage, inwiefern die genannte Krisendebatte Eingang in diesen Aushandlungsprozess findet. Krisennarrative können sich etwa in professionellen und akademischen Diskursen auswirken, an denen Studierende während ihrer Ausbildung teilhaben. Diese Wahrnehmungen prägen dann ihrerseits wiederum die künftige Auseinandersetzung über die Profession entscheidend mit.

Aus einer von der Politikwissenschaftlerin Vivien Schmidt (2008) entwickelten Spielart des Institutionalismus lässt sich Journalismus gewinnbringend als diskursive Institution beschreiben (Hanitzsch \& Vos 2016). Institutionen werden allgemein verstanden als menschengemachte Rahmenbedingungen des Handelns, die Ordnung schaffen und Unsicherheit reduzieren (North 1991). Sie beeinflussen menschliches Handeln durch die Bereitstellung von kognitiven Skripten, Kategorien und Modellen (Hall \& Taylor 1996). Der Unterschied zu traditionellen institutionalistischen Ansätzen besteht darin, dass eine diskurstheoretische Perspektive auf die Konstruktion eines manifesten Substrats verzichtet. Nach Foucault (1973) bringen Diskurse die Dinge hervor, über die sie sprechen. In diesem Verständnis verfügt Journalismus - wie auch andere soziale Institutionen - über keine wie auch immer geartete „Essenz“. Journalismus als Institution existiert, weil und indem wir über ihn sprechen.

Journalismus sowie die mit ihm verbundenen Werte, Praktiken und Kulturen sind mithin erkenn- und greifbar, da sie als Teil eines größeren Bezugsrahmens - eines Diskurses - existieren (Panizza \& Miorelli 2013). Dieser Diskurs dreht sich maßgeblich um die Deutungshoheit über die Identität und Rolle von Journalismus in der Gesellschaft. Die verschiedenen Akteure artikulieren in diesem Diskurs ihre Positionen und stehen daher miteinander in einem Wettbewerb um diskursive Autorität, wobei die diskursiven Gewichte ungleich verteilt sind. Letztlich ist der Diskurs über die Bedeutung und Rolle von Journalismus in der Gesellschaft die zentrale Arena, in der Identität und Rolle von

1 http://www.hochschulkompass.de; http://www.medienstudienfuehrer.de. 
Journalismus verhandelt werden. Journalismus als diskursive Institution repräsentiert hier den aktuellen „Verhandlungsstand“ in gesellschaftlichen Debatten über Journalismus (Hanitzsch \& Vos 2016). Dieser Diskurs vollzieht sich zudem innerhalb der relevanten sozialen Kontexte, in die Journalismus eingebettet ist. Von besonderer Relevanz sind hierbei die ökonomischen Strukturen, die Journalisten und Redaktionen mit den nötigen Ressourcen versorgen und die - wie weiter unten ausgeführt - in jüngerer Zeit vor teilweise existenziellen Herausforderungen stehen.

Als Ergebnis dieses Aushandlungsprozesses „sedimentieren“ dominante diskursive Positionen als institutionelle Normen und Regeln sowie Werte und Praktiken (Howarth 2000). Diese Strukturen stecken den Rahmen für die als „angemessen“ und „akzeptabel“ erachtete Praxis ab (Schmidt 2008). Als dynamische Struktur ist dieses Regelwerk wiederum Gegenstand von diskursiver (Re-)Kreation, (Re-)Interpretation, selektiver Aneignung und Reform. Eine zentrale Funktion dieses Diskurses besteht in der Legitimierung und Delegitimierung bestimmter professioneller Wertevorstellungen, Normen und Praktiken (Chilton \& Schäffner 2011). Journalismus stellt damit den diskursiven „Werkzeugkasten“ zur Verfügung, der es Journalisten ermöglicht, durch die Komplexitäten und Unsicherheiten ihrer Tätigkeit zu navigieren. Der Diskurs über die gesellschaftliche Identität und Rolle von Journalismus beschränkt sich dabei keinesfalls auf die journalistischen Akteure selbst. In einer Vielzahl von Filmen werden z. B. Journalisten in tragenden Rollen als Helden, Abenteurer und Aufklärer, aber auch als Schurken und Klatschreporter dargestellt (Barris 1976; Ehrlich 2004).

Die Journalistenausbildung befindet sich an der Schnittstelle zwischen professionellen und akademischen Diskursen sowie einer breiteren gesellschaftlichen Auseinandersetzung. Über die berufliche Sozialisation wachsen junge Nachwuchsjournalisten allmählich in eine diskursive Gemeinschaft hinein. Dieser Prozess vollzieht sich sowohl in redaktionellen Organisationen als auch während der beruflichen Aus- und Weiterbildung (Gravengaard \& Rimestad 2014). Hier werden nachwachsende Journalistengenerationen in eine Kultur eingeführt, in der journalistische „Veteranen“ die Standards definieren und kollektive Mythen darüber forterzählen, was gemeinhin als „guter Journalismus" gehandelt wird. Zentral sind hierbei journalistische Schlüsselmomente, wie etwa die Aufdeckung des Watergate-Skandals in den USA, die aus der kollektiven Vorstellungswelt des amerikanischen Journalismus nicht wegzudenken sind (Zelizer 1993).

Diese Vorstellungswelt und die damit verbundenen gesellschaftlichen Diskurse sind durch aktuelle - vor allem ökonomische und technologische-Entwicklungen nachhaltig erschüttert worden. Hier zeigt sich das Gesicht der Krise vor allem auf sechs Ebenen: (1) das bröckelnde journalistische Geschäftsmodell, (2) die veränderten Mediennutzungsgewohnheiten, (3) der Einfluss partizipativer Technologien, (4) der Funktionswandel im Journalismus, (5) sein öffentlicher Reputationsverlust sowie - in der Konsequenz - (6) die Veränderung des beruflichen Arbeitsumfelds.

- Ad (1) Auf ökonomischer Ebene gerät das traditionelle Geschäftsmodell des Journalismus zunehmend unter Druck. Als Hauptgründe hierfür werden sinkende Auflagen von Tageszeitungen sowie ein zum Teil dramatischer Rückgang der Anzeigenerlöse ausgemacht (Meier 2009; Röper 2012). Bei genauerer Betrachtung wird allerdings deutlich, dass es sich bei den aktuellen Entwicklungen tatsächlich um eine Tageszeitungsfinanzierungskrise handelt (Jarren 2012). Tageszeitungen verlieren auf dem Werbemarkt kontinuierlich Marktanteile; Anzeigen wandern zunehmend ins Netz (BDZV 2012).

- Ad (2) Hierzu trägt eine veränderte Mediennutzung erheblich bei. Während die Reichweite der Tageszeitungen unter den über 60 Jahre alten Menschen noch bei 
knapp 80 Prozent liegt, verliert das Medium anhaltend junge Leser (BDZV 2012; Meier 2009). Jüngere Lesergenerationen steigen auf mobile Angebote um, die nach wie vor günstig und schnell verfügbar sind.

- Ad (3) Partizipative Technologien im Internet - insbesondere die sozialen Medien stellen traditionelle Medienangebote vor neue Herausforderungen (Singer et al. 2011). Mit dem Internet steht als Alltagsmedium ein Kommunikationskanal zur Verfügung, der Journalismus in seiner exklusiven Funktion als Bereitsteller aktueller Informationen existenziell herausfordert (Lünenborg 2012).

- Ad (4) Damit einher geht ein Funktionswandel von Journalismus - vom „Gatekeeper" zum "Gatewatcher" (Bruns 2005). Da der Zugang zu Information in der digitalen Welt theoretisch unbegrenzt ist, verändert sich auch die gesellschaftliche Rolle von Journalismus. Im Vordergrund steht heute vermehrt eine Navigationsfunktion: Journalismus ordnet und verifiziert Informationen und bietet Orientierung in einer zunehmend multioptionalen Gesellschaft (Rössler, Hautzer \& Lünich 2014).

- Ad (5) Dabei scheint die öffentliche Reputation und das Vertrauen in journalistische Nachrichtenangebote zu schwinden. Donsbach et al. (2009) sprechen in diesem Zusammenhang von der „Entzauberung eines Berufs“. In der Allensbacher Berufsprestige-Skala rangieren Journalisten seit längerer Zeit auf den hinteren Plätzen (IfD Allensbach 2013); Medien werden auf Montagsdemonstrationen pauschal als „Lügenpresse“ beschimpft. Nach einer Allensbach-Studie fühlt sich eine Mehrheit der Deutschen über Flüchtlinge einseitig informiert (Köcher 2015). ${ }^{2}$

- Ad (6) Als Konsequenz hat sich das journalistische Arbeitsumfeld nachhaltig verändert. Medienunternehmen sparen beim Personal, streichen feste Stellen und setzen vermehrt auf freie Journalisten, Pauschalisten und Leihredakteure (vgl. DJV 2009; Schnedler 2013). Das Einkommen der freien Journalisten liegt bei deutlich weniger als der Hälfte dessen, was angestellte Redakteure verdienen (DJV 2014).

Es überrascht wenig, dass Journalisten vor diesem Hintergrund ihre berufliche Zukunft oft düster sehen. In einer Umfrage unter knapp 1700 Journalisten haben 54 Prozent der Befragten angegeben, sie würden ihre Situation als „sehr" oder „eher schwierig" bewerten (Marketagent 2010). In der gleichen Studie befanden 55 Prozent der Journalisten ihre Arbeitsbedingungen für „,weniger“ oder ,überhaupt nicht gut“. Und über zwei Drittel der Befragten waren der Meinung, dass zahlreiche Tageszeitungen im Jahr 2020 bereits nicht mehr erscheinen werden. Greck und Altmeppen (2012) betiteln einen Aufsatz zum Journalistenberuf daher pointiert mit „Traumberuf oder Berufstraum(a)?“

\section{Diskursives Echo: Einstellungen und Motivation von Journalismus- Studierenden}

Der Journalismus steht also vor umfassenden Herausforderungen, und die Krisendebatten verheißen Journalismus-Studierenden keine rosige Zukunft. Wir wissen zurzeit jedoch noch recht wenig darüber, wie sich diese Entwicklungen in den Sichtweisen der Studierenden niederschlagen. In privatwirtschaftlich betriebenen Ausbildungsinstitutionen macht sich die schlechte Arbeitsmarktlage jedenfalls seit geraumer Zeit bemerkbar (Neuberger 2005). Von Beteiligten wird hinter vorgehaltener Hand von einem schleichenden Rückgang der Bewerberzahlen bei entsprechenden Studiengängen berichtet.

2 Die gleiche Studie zeigt aber auch ein weitverbreitetes Grundvertrauen. Mehr als zwei Drittel der Bevölkerung halten die Berichte des öffentlich-rechtlichen Fernsehens und der Tagespresse im Allgemeinen für zuverlässig. Ähnliche Ergebnisse zeigt ein Blick in Zeitreihendaten des European Social Survey und des World Values Survey. 
Zur akuten Problematik kommt erschwerend hinzu, dass die hochschulgebundene Journalistenausbildung in Deutschland in der Praxis oft wenig Anerkennung findet (Harnischmacher 2010b). Unvergessen bleibt Detlef Esslingers (2009) Abrechnung mit der Journalistik als „Leerfach“. Der SZ-Redakteur riet darin angehenden Journalisten, anstatt Journalismus „ein Fach von Belang“ zu studieren. Denn: Wer Journalistik studiere, sei „anschließend Fachmann in nichts“. Harnischmacher (2010a) kommt in einer Befragung von Chefredakteuren zu dem Ergebnis, dass eine Mehrheit (56 \%) einschlägige Studiengänge für ungeeignet hält - sofern sie nicht mit einem Volontariat kombiniert werden. Gleichzeitig aber attestiert Neuberger (2010) auf Basis einer Alumnibefragung den Absolventen der Kommunikations- und Medienwissenschaft gute Berufschancen. Beim Punkt Berufseinstieg hätten vor allem Journalistik-Absolventen die besten Karten (Neuberger 2005).

Viele Befragungen von Studierenden und Absolventen deutscher Journalismus-Studiengänge sind jedoch institutionengebunden und lassen nur in der Tendenz Aussagen über die Situation in Deutschland zu (Neuberger 2002; Westerbarkey \& Winkelbrandt 1996). In den USA hingegen gibt es systematische und vergleichende Studien bereits seit den 1970er und verstärkt seit den 1980er Jahren (Becker, Fruit \& Caudill 1987; Bowers 1974). Komparative Untersuchungen zu diesem Thema haben gerade in jüngerer Zeit einen rasanten Aufschwung erlebt (Hovden et al. 2009; Mellado et al. 2013; Nygren, Degtereva \& Pavlikova 2010; Sanders et al. 2008). Aus den Ergebnissen der mit 22 Ländern bislang umfangreichsten Studie folgerten Splichal und Sparks (1994) einen weltweiten Trend zur Professionalisierung. Mehrere vergleichende Untersuchungen konnten zudem zeigen, dass Nachwuchsjournalisten bereits während ihrer vorberuflichen Sozialisation durch die jeweiligen „nationalen Journalismuskulturen“ nachhaltig beeinflusst werden (Sanders et al. 2008), was die Unterschiede in ihren professionellen Einstellungen erklärt (Mellado et al. 2013).

Laut einer Untersuchung von Nygren et al. (2010) blickt etwa ein Drittel der russischen Journalismus-Studierenden pessimistisch in die Zukunft. Bei ihren schwedischen Kommilitonen war es hingegen nur ein Fünftel. In beiden Ländern werden Medien als zu kommerziell und zu unterhaltungsorientiert eingeschätzt. In einer Erweiterung der Studie auf Russland, Polen, Schweden, Estland und Finnland konnten Nygren und Stigbrand (2014) fünf Faktoren identifizieren, von denen nach Meinung der befragten Studierenden die stärksten Bedrohungen für den Journalismus ausgehen: Profiterwartungen der Unternehmen, politische Beeinflussung, das gestiegene Arbeitstempo, die Konzentration von Medieneigentum sowie der Einfluss der Werbeindustrie.

In wirtschaftlich schwierigen Zeiten sehen zudem längst nicht alle Studierenden ihre Zukunft im Journalismus. So waren sich nur 58 bzw. 42 Prozent der Studierenden in Dänemark und Norwegen „absolut sicher“, nach dem Studium in den Journalismus zu gehen (Hovden et al. 2009). In Finnland und Schweden lag dieser Anteil sogar bei nur 18 bzw. 30 Prozent. In der bereits erwähnten Fünf-Länder-Studie von Nygren und Stigbrand (2014) streute der Wert in einem relativ engen Korridor zwischen 26 und 35 Prozent.

Hoher Konkurrenzdruck und finanzielle Unsicherheiten können die Vorstellungen der Studierenden dabei verändern oder sie von ihrem Berufswunsch sogar abbringen. Hanna und Sanders (2007) berichten für britische Journalismus-Studierende, dass der Teil jener, die „ganz sicher" in den Journalismus wollten, im Laufe des Studiums von anfänglich 75 auf schließlich 53 Prozent zurückging (zusätzlich zu den ca. 15 \% Studienabbrechern). Bei einer Befragung von Journalismus-Studierenden in Norwegen haben 
Bjørnsen, Hovden und Ottosen (2007) festgestellt, dass der anfängliche Idealismus im Laufe der Ausbildung und der frühen beruflichen Sozialisation langsam erodiert.

Umfassend untersucht ist zudem, was junge Menschen zu einem Studium der Journalistik bzw. des Journalismus motiviert. Hierbei werden allgemein interne und externe Faktoren unterschieden (Splichal \& Sparks 1994) - bzw. nach modernerer Lesart: intrinsische und extrinsische Motivationen (Wagner 2009). Die vorliegenden Studien deuten für Journalismus-Studierende übereinstimmend auf eine stärker intrinsische $-\mathrm{d}$. $\mathrm{h}$. innere, aus sich selbst heraus entstehende-Motivation hin. Studierende versprechen sich vom Journalismus dabei eine abwechslungsreiche und unkonventionelle Tätigkeit, bei der sie viel mit Menschen zu tun haben. Darüber hinaus verbinden sie mit dem Beruf die Freiheit und Unabhängigkeit, an interessanten Themen zu arbeiten und dabei ihre Kreativität und Freude am Schreiben auszuleben (Hanna \& Sanders 2007; Hovden et al. 2009; Nygren \& Stigbrand 2014; Sanders et al. 2008). Nygren und Stigbrand (2014) führen diese Motive zu einer Kategorie „Selbstverwirklichung“ zusammen, die sie zwei weiteren Motivgruppen gegenüberstellen: „soziale Reform“ und „Geld und Status“.

Der vorliegende Beitrag möchte folglich untersuchen, welchen Schatten die aktuelle Krise im Journalismus auf die Einstellungen und Motivation von deutschen Journalismus-Studierenden wirft. Konkret geht es dabei um folgende Forschungsfragen:

- Wie bewerten die Studierenden die Zukunftschancen des Journalismus?

- Wo sehen sie die größten Herausforderungen bzw. Probleme für den Journalismus in Deutschland?

- Welche Aspekte haben sie zu einem Studium der Journalistik bzw. des Journalismus motiviert?

- Streben die Studierenden tatsächlich eine Karriere als Journalistin bzw. Journalist an und in welchen Medienbereichen möchten sie arbeiten?

- Wie optimistisch bzw. pessimistisch sehen sie in ihre eigene berufliche Zukunft?

- Haben sich Karriereziele und Zukunftseinschätzung im Laufe des Studiums verändert?

\section{Methodische Anlage}

Im Vorfeld der Befragung wurden zunächst die Literatur und einschlägige Datenbanken konsultiert, um einen Überblick über die hochschulgebundene Journalistenausbildung in Deutschland zu erhalten. Hilfreich waren verschiedene Überblicksarbeiten (u. a. Harnischmacher 2010a) sowie die Studienführer von Burgard und Schröder (2012) und Henning und Henning (2010). Anhand der online verfügbaren Daten aus dem Hochschulkompass und Medienstudienführer ${ }^{3}$ wurden die Listen schließlich aktualisiert. Um in die Auswahl zu gelangen, musste die Bezeichnung "Journalistik“ oder "Journalismus“ im Namen des Studiengangs enthalten sein (bzw. „Redakteur“ oder „Redaktion"). Rein medien- oder kommunikationswissenschaftliche Studiengänge wurden nicht berücksichtigt. Im Frühjahr 2014 zählten wir anhand dieser Informationen insgesamt 24 einschlägige grundständige und 22 Master-Studiengänge an neun Universitäten und 21 Fachhochschulen. Akademien sowie Kunst- und Musikhochschulen sind hierbei nicht berücksichtigt.

Aktuelle Journalismus-Studierendenzahlen sind schwer zu erheben, da sie in den Angaben des Bundesamts für Statistik nicht mehr separat von Studierenden der Kommunikationswissenschaft ausgewiesen werden. Die letzten Zahlen stammen aus dem

3 http://www.hochschulkompass.de; http://www.medienstudienfuehrer.de. 
Wintersemester 2007/08: Zu dieser Zeit waren insgesamt 2400 Journalismus-Studierende an diversen Hochschulen im Bundesgebiet eingeschrieben. Die Zahl der Studienanfänger bewegte sich im Zeitraum von 1998 bis 2007 auf einem Niveau zwischen 258 (im Jahr 2003) und 402 Studierenden (2002). Im Durchschnitt waren es 314 Personen, die in diesem Zeitraum ihr Studium des Journalismus bzw. der Journalistik angetreten hatten.

Für die vorliegende Studie wurde die Stichprobe aus ressourcentechnischen Gründen reduziert. Die zu berücksichtigenden Vollzeitstudiengänge sollten bereits seit mindestens drei Jahren operieren, mindestens 30 Studierende umfassen und grundlegende Kernkompetenzen im Journalismus vermitteln. Darüber hinaus wurde darauf geachtet, dass die Auswahl die thematische und geografische Vielfalt der hochschulgebundenen Journalistenausbildung angemessen widerspiegelt. Auf diese Weise wurden insgesamt 15 relevante Standorte - Universitäten und Fachhochschulen - identifiziert. Im nächsten Schritt wurden diese Standorte um ihre Mitwirkung gebeten. Dabei wurde - soweit möglich - auf bestehende Arbeitskontakte zurückgegriffen. Von den kontaktierten Einrichtungen erklärten sich schließlich elf Ausbildungseinrichtungen zur Mitarbeit bereit. Die verbleibenden Hochschulen reagierten nicht auf unsere Anfragen oder verweigerten explizit die Teilnahme (u. a. aus Gründen des Datenschutzes). In der Tendenz handelte es sich hierbei vor allem um Einrichtungen, die kaum oder gar nicht in die wissenschaftliche Community eingebunden sind.

Wir glauben dennoch, dass die Studie das Universum der deutschen Journalismusbzw. Journalistik-Studierenden hinreichend gut abbilden kann. Durch den Ausschluss von Spartenstudiengängen wie „Kreatives Schreiben und Kulturwissenschaft“ der Universität Hildesheim wird jedoch deutlich, dass sich die vorliegende Studie insbesondere dem traditionellen Kernbereich des Journalismus zuwendet, in dem die überwiegende Mehrheit der Journalisten tätig ist. ${ }^{4} \mathrm{Zu}$ den teilnehmenden elf Institutionen zählten folgende Hochschulen: BiTS Iserlohn, Hochschule Bremen, Hochschule Darmstadt, Hochschule Macromedia, Jade Hochschule, Katholische Universität Eichstätt-Ingolstadt, LMU München, Technische Universität Dortmund, Universität Hamburg, Universität Leipzig sowie Universität Mainz (siehe Übersicht im Anhang). Nach Auskunft der dortigen Ansprechpartner waren an diesen Einrichtungen zum Zeitpunkt der Befragung insgesamt etwa 1500 Studierende in Studiengängen der Journalistik bzw. des Journalismus eingeschrieben.

Die Befragung selbst wurde im Sommersemester 2014 gemeinsam mit Studierenden des Master-Studiengangs „Journalismus“ an der LMU München als Lehrprojekt realisiert. Das Projekt war dabei eingebettet in die zurzeit laufende länderübergreifende Vergleichsstudie "Journalism Students around the Globe“, für die Studierendenbefragungen in etwa 30 Ländern international koordiniert werden. Zur Gewährleistung der Vergleichbarkeit hatten sich die Projektpartner auf ein einheitliches Vorgehen und einen gemeinsamen Fragebogen verständigt. Aus diesem Grund war bei der inhaltlichen und technischen Gestaltung des Fragebogens (sowie auch des Stichprobendesigns) nur bedingte Flexibilität gegeben.

Für die Studie wurden von April bis Juni 2014 postalische Befragungen an den oben genannten Hochschulen in Deutschland durchgeführt. Hierfür wurde eine jeweils vereinbarte Anzahl von Fragebögen an die elf teilnehmenden Ausbildungseinrichtungen geschickt. Die Zahl der Fragebögen orientierte sich an dem Anteil der Studierenden, die für die Befragung vor Ort erreichbar waren. Die Verteilung der Fragebögen an die Stu-

4 An diesem Studiengang werden laut Modulhandbuch Kernkompetenzen und handwerkliche Elemente des Journalismus wie z. B. die Recherche nicht vermittelt. 
dierenden wurde über die dortigen Ansprechpartner ${ }^{5}$ realisiert. Die ausgefüllten Fragebögen wurden anschließend über die Ausbildungsinstitutionen an die Projektleitung zurückgesendet. Als zusätzliche Incentives, um die Studierenden zur Teilnahme zu motivieren, wurden nach der Befragung insgesamt zehn Wertgutscheine in Höhe von 25 Euro verlost. 1300 Fragebögen sind an die teilnehmenden Einrichtungen gegangen, von denen 556 schließlich für die Studie verwertbar waren. Die Ausschöpfung lag somit bei reichlich 50 Prozent.

\section{Die Stichprobe: mehrheitlich weiblich, praxiserfahren und akademisches Milieu}

Die Studierenden in der Stichprobe waren mehrheitlich weiblich (63,1 \%) und im Mittel 22,2 Jahre alt (Median: 22 Jahre). Vier von fünf Befragten studierten in einem grundständigen Studiengang (Bachelor of Arts, 79,7\%). Der überwiegende Teil der Teilnehmer war im ersten bzw. zweiten Fachsemester (57,7\%), ein weiteres knappes Drittel im dritten oder vierten Semester (31,7\%). Unter den 113 Befragten, die in einem MasterStudiengang eingeschrieben waren, befanden sich insgesamt 43 Personen mit einem medien-, kommunikations- oder publizistikwissenschaftlichen Abschluss bzw. immerhin 19 Studierende mit einem BA in Journalismus oder einem verwandten Fach.

Eine überwältigende Mehrheit der befragten Studierenden verfügte zum Zeitpunkt der Untersuchung bereits über einschlägige Arbeitserfahrung (92,4 \%). Im Durchschnitt lag diese Zeit bei insgesamt 14 Monaten; 55,1 Prozent konnten dabei mindestens sechs Monate Erfahrung vorweisen. Über die Hälfte der Studienteilnehmer stammt aus einem akademischen Elternhaus (52,6 \%); in 27,2 Prozent der Fälle verfügten beide Eltern über einen Hochschulabschluss. Unter den 499 Studierenden, die den Beruf ihrer Eltern genannt haben, fanden sich zehn Befragte, deren Eltern auch Journalisten waren. In zwei Fällen waren sogar beide Eltern im Journalismus tätig.

Um eine differenzierte Bewertung der Einstellungen von Studierenden in Abhängigkeit von der Studiendauer zu ermöglichen, wurden anhand der Angaben zum Studienjahr zwei Gruppen gebildet: Eine Gruppe bestand aus Studierenden im ersten Studienjahr (55,2 \%), wohingegen die zweite Gruppe aus Studierenden in höheren Semestern bestand. Master-Studierende mit einem BA-Vorstudium in Journalismus wurden grundsätzlich der zweiten Gruppe zugerechnet. In ähnlicher Weise wurden drei Gruppen nach dem Umfang der praktischen Erfahrung gebildet: keine Berufserfahrung, bis zu sechs Monaten Praxis sowie mehr als sechs Monate Berufserfahrung.

\section{Zukunftschancen des Journalismus und seine größten Herausforderungen}

Die Zukunftschancen des Journalismus in Deutschland bewertet die Mehrheit der Studierenden ambivalent. Insgesamt 64,7 Prozent der Befragten gaben an, „weder optimistisch noch pessimistisch" in die Zukunft des Journalismus zu schauen. Gleichzeitig aber ist der Anteil der Studierenden, die eher pessimistisch eingestellt sind, mit 23,9 Prozent um einiges größer als jener der Optimisten (11,5\%). Der Anteil der Pessimisten unter den Studierenden im ersten Studienjahr lag mit 21,5 Prozent nur geringfügig unter dem entsprechenden Anteil für die „Fortgeschrittenen“ (26,3 \%), während der Prozentsatz der „Ambivalenten“ unverändert blieb. Gleichzeitig verringert sich der Anteil der Optimisten mit der Berufserfahrung: Unter Studierenden ohne Praxiserfahrung liegt dieser Anteil noch bei 17,4 Prozent, er schrumpft bei Befragten mit bis zu sechs Monaten Berufspraxis auf 12,1 Prozent und bei jenen mit mehr als sechs Monaten auf 8,8 Prozent.

5 Unseren Ansprechpartnern möchten wir an dieser Stelle herzlich danken. 
In beiden Fällen aber sind die Unterschiede nicht signifikant. Die Bewertung der Zukunftschancen von Journalismus scheint sich dabei auch unmittelbar auf die Karrierepläne der Studierenden auszuwirken. Der Anteil der Pessimisten ist mit 16,5 Prozent unter angehenden Journalisten deutlich und signifikant geringer als unter denjenigen Befragten, die sicher nicht in den Journalismus wollen $(36,7 \%){ }^{6}$

Insgesamt lässt sich also feststellen, dass es unter den befragten Studierenden keinen ausgeprägten Pessimismus hinsichtlich der Zukunftschancen des Journalismus zu beobachten gibt, auch wenn etwa ein Viertel von ihnen die Zukunft eher negativ beurteilt. Die Ergebnisse decken sich mit der schwedischen Studie von Nygren et al. (2010). Der große Anteil der „Ambivalenten“ ist vermutlich Ausdruck der aktuellen Situation, in der nicht ganz klar ist, welchen Weg der Journalismus in der Zukunft beschreiten wird.

Die größten Herausforderungen bzw. Probleme für den Journalismus in Deutschland sehen die Studierenden vor allem in ökonomischen Faktoren sowie in verschlechterten Arbeitsbedingungen. Wie Tabelle $1 \mathrm{zu}$ entnehmen ist, geht für mehr als die Hälfte der Befragten eine „große“ bzw. „extreme Gefahr“ von folgenden Problemen aus: unzureichenden finanziellen Ressourcen, Gewinnerwartungen von Investoren, der Beschleunigung von Arbeitsabläufen, der Marktorientierung im Journalismus, dem Einfluss von Werbung sowie der Konzentration von Medieneigentum. Auch hier liegen die deutschen Studierenden auf einer Linie mit ihren Kommilitonen in Finnland und Schweden sowie in Estland, Polen und Russland (Nygren \& Stigbrand 2014).

Einiges Gefährdungspotenzial geht nach Auffassung der Studierenden aber auch von externen Kräften aus, die versuchen, Journalisten für ihre Zwecke zu benutzen (Öffentlichkeitsarbeit und Lobby-Gruppen), sowie vom zunehmenden Wettbewerb mit dem Internet, einer Verschränkung von Medien und Politik, staatlichen Eingriffen in die Medien und einem eingeschränkten Zugang zu Information. Andere Gefahrenquellen, die eher aus dem Journalismus selbst heraus generiert werden, sind eine unzureichende professionelle Ethik, parteiischer Journalismus und eine steigende Abhängigkeit von freien Journalisten. Wenig Sorge bereitet den Studierenden in Deutschland hingegen die Zensur und Selbstzensur, die Qualität der Journalistenausbildung, der allgemeine gesetzliche Kontext sowie ausländische Investitionen in deutsche Medienunternehmen.

\section{Motive für die Studienwahl}

Eine zentrale Fragestellung der vorliegenden Studie waren die Motive der Studierenden für ein Studium der Journalistik bzw. des Journalismus. Hierfür wurde den Befragten ein Katalog von insgesamt 19 Motiven vorgelegt, deren Wichtigkeit für die Studienwahl auf einer fünfstufigen Skala eingeschätzt werden sollte. Die in Tabelle 2 berichteten Ergebnisse korrespondieren im Großen und Ganzen mit Resultaten aus ähnlichen Studien in anderen Ländern (Hanna \& Sanders 2007; Hovden et al. 2009; Nygren \& Stigbrand 2014; Sanders et al. 2008). Junge Menschen entscheiden sich für ein Journalismus-Studium aufgrund von Motiven, die sich überwiegend der Kategorie „Selbstverwirklichung“ zurechnen lassen (Nygren \& Stigbrand 2014).

Journalismus bezieht seine Attraktivität demnach aus der damit verbundenen Möglichkeit, einer abwechslungsreichen und aufregenden Tätigkeit nachzugehen, unterschiedliche Menschen kennenzulernen sowie die eigene Kreativität, Freude am Schreiben und das journalistische Talent auszuleben. Darüber hinaus schätzen die Studierenden

6 Der Anteil der Optimisten ist dabei relativ unverändert (Berufsziel Journalismus: 11,8 \%; andere: $10,0 \%)$. Allerdings fanden sich unter angehenden Journalisten mehr „Ambivalente“ (71,7 vs. $53,3 \%) \cdot \chi^{2}(4)=32,68 ; \mathrm{p}<0.001$ 
Tabelle 1: Probleme, von denen eine Gefahr für den Journalismus ausgeht

\begin{tabular}{lcccc}
\hline & $N$ & $\begin{array}{c}\text { "große“ und } \\
\text { "extreme } \\
\text { Gefahr }\end{array}$ & $\begin{array}{c}\text { Mittelwert } \\
\text { Standard- } \\
\text { abweichung }\end{array}$ \\
\hline Unzureichende finanzielle Ressourcen & 543 & $71,5 \%$ & 3,91 &, 91 \\
Gewinnerwartungen der Investoren & 542 & $64,8 \%$ & 3,73 &, 87 \\
Beschleunigung der Arbeitsabläufe & 542 & $64,0 \%$ & 3,74 &, 98 \\
Marktorientierung im Journalismus & 543 & $62,4 \%$ & 3,71 &, 87 \\
Einfluss der Werbung auf den Inhalt & 542 & $52,6 \%$ & 3,49 &, 94 \\
Konzentration von Medieneigentum & 544 & $50,6 \%$ & 3,45 &, 84 \\
PR, Öffentlichkeitsarbeit und Lobby-Gruppen & 543 & $49,7 \%$ & 3,48 &, 97 \\
Unzureichende professionelle Ethik & 544 & $49,2 \%$ & 3,46 & 1,01 \\
Wettbewerb mit dem Internet & 544 & $44,3 \%$ & 3,28 & 1,12 \\
Parteiischer Journalismus & 544 & $40,3 \%$ & 3,23 &, 99 \\
Verschränkung von Medien und Politik & 540 & $38,1 \%$ & 3,24 &, 86 \\
Staatliche Eingriffe in die Medien & 547 & $37,7 \%$ & 3,13 & 1,01 \\
Eingeschränkter Zugang zu Information & 546 & $37,2 \%$ & 3,11 & 1,04 \\
Steigende Abhängigkeit von freien Journalisten & 543 & $36,3 \%$ & 3,06 & 1,10 \\
Unzureichende Berufsausbildung & 543 & $28,5 \%$ & 2,89 & 1,02 \\
Zensur & 546 & $26,2 \%$ & 2,71 & 1,22 \\
Physische Bedrohungen gegen Journalisten & 547 & $25,2 \%$ & 2,76 & 1,11 \\
Selbstzensur & 544 & $21,7 \%$ & 2,79 &, 95 \\
Die Qualität der Journalistenausbildung & 545 & $20,4 \%$ & 2,62 & 1,01 \\
Gesetzliche Regelungen & 542 & $18,1 \%$ & 2,73 &, 88 \\
Ausländische Investitionen in deutsche Medien & 541 & $14,2 \%$ & 2,62 &, 89 \\
\hline
\end{tabular}

„Was sind Ihrer Meinung nach die größten Herausforderungen bzw. Probleme für den Qualitätsjournalismus in Deutschland? Bitte geben Sie an, in welchem Ausmaß Sie die folgenden Aspekte als Gefahr ansehen."

Skala: 1=„keine Gefahr“; 2=,,geringe Gefahr“; 3=„teilweise Gefahr“; 4=,,große Gefahr“; 5=,,extreme Gefahr".

die mit dem Beruf verbundene Unabhängigkeit. Idealistische Motive wie die Bekämpfung von Ungerechtigkeit, Hilfestellung im Alltag sowie das Eintreten für Freiheit und Demokratie befinden sich entweder im Mittelfeld oder sortieren sich weiter hinten ein.

Angesichts der schwierigen Arbeitsmarktlage und Anstellungsbedingungen im Journalismus ist es wenig überraschend, dass materielle Überlegungen wie Verdienstmöglichkeiten und Arbeitsplatzsicherheit in der Rangfolge der Motive eher weiter unten stehen. Den Studierenden ist durchaus bewusst, dass der Journalismus kein Beruf ist, in dem hohe Verdienste und große Sicherheiten zu erwarten sind. Das gesellschaftliche Ansehen von Journalismus war zudem für weniger als 18 Prozent der Befragten ein wichtiges Motiv für die Studienwahl.

Dabei ist eine leichte Verschiebung in der Motivbewertung in Abhängigkeit von der Berufserfahrung zu beobachten. Die Bedeutung von einigen idealistischen Motiven und jenen, die auf Selbstverwirklichung gerichtet sind, nimmt in der Tendenz zu, wohingegen die Relevanz von materiellen Motiven zurückgeht. Ein Vergleich der Mittelwerte zeigt einen signifikanten Bedeutungsgewinn für die Motive „unterschiedliche Menschen kennenlernen“, „Freude am Schreiben“, „Talent für den Journalismus“, „gegen Ungerechtigkeit vorgehen“, „für Freiheit und Demokratie eintreten“ und „die Mächtigen in die 
Verantwortung nehmen ". ${ }^{7}$ Gleichzeitig verlieren die Verdienstmöglichkeiten und Arbeitsplatzsicherheit an Motivationskraft für ein Studium des Journalismus. ${ }^{8}$ Es scheint also, als würden sich die Studierenden mit zunehmender Praxiserfahrung von materiellen Motiven eher verabschieden und den Sinn ihrer beruflichen Tätigkeit stärker in der Selbstverwirklichung und im gesellschaftspolitischen Idealismus suchen. Dieser Befund schlägt sich auch beim Gruppenvergleich nieder: Diejenigen, die als Berufsziel Journalismus angegeben haben, ließen sich deutlich weniger von antizipierten Verdienstmöglichkeiten (Mittelwerte: 2,48 vs. 3,03) und vermuteter Arbeitsplatzsicherheit (Mittelwerte: 2,23 vs. 2,85 ) leiten. ${ }^{9}$

\section{Tabelle 2: Motive für Studienantritt}

\begin{tabular}{lcccc}
\hline & $N$ & $\begin{array}{c}\text { "sehr" und } \\
\text { "extrem } \\
\text { wichtig “ }\end{array}$ & Mittelwert & $\begin{array}{c}\text { Standard- } \\
\text { abweichung }\end{array}$ \\
\hline Abwechslungsreiche und aufregende Tätigkeit & 552 & $94,0 \%$ & 4,43 &, 63 \\
Kreativ sein & 554 & $87,0 \%$ & 4,25 &, 77 \\
Unterschiedliche Menschen kennenlernen & 553 & $80,3 \%$ & 4,08 &, 84 \\
Freude am Schreiben & 551 & $73,1 \%$ & 3,95 & 1,04 \\
Talent für den Journalismus & 551 & $56,3 \%$ & 3,52 &, 98 \\
Grad an Unabhängigkeit & 552 & $52,2 \%$ & 3,46 &, 96 \\
Dynamischer Lebensstil & 553 & $49,7 \%$ & 3,37 & 1,07 \\
Gegen Ungerechtigkeit vorgehen & 553 & $47,4 \%$ & 3,32 & 1,06 \\
Menschen bei alltäglichen Problemen helfen & 551 & $37,4 \%$ & 3,11 & 1,01 \\
Für Freiheit und Demokratie eintreten & 551 & $36,5 \%$ & 3,11 & 1,07 \\
Reisen & 550 & $30,9 \%$ & 2,81 & 1,13 \\
Die Mächtigen in die Verantwortung nehmen & 549 & $29,5 \%$ & 2,88 & 1,05 \\
Verdienstmöglichkeiten & 554 & $22,0 \%$ & 2,68 & 1,03 \\
Politik beeinflussen & 552 & $21,7 \%$ & 2,67 & 1,02 \\
Arbeitsplatzsicherheit & 550 & $18,5 \%$ & 2,45 & 1,13 \\
Ansehen von Journalismus als Beruf & 552 & $17,9 \%$ & 2,53 & 1,06 \\
Regierung helfen, Deutschland voranbringen & 551 & $6,7 \%$ & 2,03 &, 94 \\
Berühmt sein & 553 & $6,5 \%$ & 1,96 &, 95 \\
Nation zusammenhalten & 548 & $5,3 \%$ & 2,02 &, 91 \\
\hline
\end{tabular}

„Bitte sagen Sie uns, inwiefern Sie die folgenden Aspekte zu Ihrem aktuellen Studium motiviert haben."

Skala: 1=„unwichtig“; 2=„,weniger wichtig“; 3=„mittel wichtig“; 4= „sehr wichtig“; $5=$ „extrem wichtig“.

7 Mittelwerte und F-Tests für jeweils drei Gruppen (keine Praxis, max. 6 Monate Praxis und mehr als 6 Monate Praxis: „unterschiedliche Menschen kennenlernen" ( $M W=3,87 / 4,00 / 4,20$; $\mathrm{F}(2 ; 533)=5,20 ; \mathrm{p}<0,01) ;$,Freude am Schreiben“ (MW=3,47/4,01/4,00; $\mathrm{F}(2 ; 532)=5,64 ; \mathrm{p}<0,01)$; „Talent für den Journalismus“ (MW=2,93/3,48/3,68; $\mathrm{F}(2 ; 531)=11,75 ; \mathrm{p}<0,001)$; ,gegen Ungerechtigkeit vorgehen “ $(\mathrm{MW}=2,93 / 3,26 / 3,41 ; \mathrm{F}(2 ; 533)=4,43 ; \mathrm{p}<0,05)$; „für Freiheit und Demokratie eintreten“ (MW=2,82/3,01/3,24; $\mathrm{F}(2 ; 531)=4,58 ; \mathrm{p}<0,05)$; „die Mächtigen in die Verantwortung nehmen" (MW=2,44/2,84/2,98; $\mathrm{F}(2 ; 529)=5,35 ; \mathrm{p}<0,01)$.

8 Mittelwerte und F-Tests: „Verdienstmöglichkeiten“ (MW=3,09/2,85/2,40; $F(2 ; 534)=16,56$; $\mathrm{p}<0,001)$; „Arbeitsplatzsicherheit“ (MW=3,07/2,63/2,15; $\mathrm{F}(2 ; 530)=19,95 ; \mathrm{p}<0,001)$.

9 T-Tests: „Verdienstmöglichkeiten“ $(\mathrm{t}(545)=6,21 ; \quad \mathrm{p}<0,001) ; \quad$ "Arbeitsplatzsicherheit “ $(\mathrm{t}(542)=6,42 ; \mathrm{p}<0,001)$. 


\section{Karriereziel und Einschätzung der eigenen beruflichen Zukunft}

Streben junge Menschen, die in einem Journalismus- bzw. Journalistik-Studiengang eingeschrieben sind, tatsächlich eine Karriere als Journalistin bzw. als Journalist an? Auf die Frage, in welchem Tätigkeitsbereich sie nach Abschluss des Studiums am liebsten arbeiten möchten, nannten nur 63,8 Prozent der befragten Studierenden den Journalismus. Weitere 13,7 Prozent nannten die Bereiche PR/Unternehmenskommunikation und 6,6 Prozent die Werbung als Berufsziel. Ein geringer Anteil war sich in dieser Frage noch nicht sicher $(11,5 \%)$.

Ähnliche Ergebnisse produzierte eine weitere Frage, die hier konkret nachfasste. Darin wurden die Befragten gebeten einzuschätzen, wie wahrscheinlich es ihrer Meinung nach ist, dass sie nach Abschluss der Hochschulausbildung als Journalistin bzw. Journalist arbeiten werden. Aus Tabelle 3 geht hervor, dass weniger als ein Viertel der Befragten der Auffassung war, dass dies „auf jeden Fall“ so kommen würde. Ein weiteres reichliches Drittel gab als Antwort „wahrscheinlich“ an. Die positive Botschaft also ist: Trotz der schwierigen Zeiten möchte immer noch eine deutliche Mehrheit der Studierenden in den Journalismus. Allerdings liegt der Anteil derer, die sich hier sehr sicher waren, im europäischen Vergleich eher am unteren Ende (Hovden et al. 2009; Nygren \& Stigbrand 2014).

Tabelle 3: Karriereziel Journalismus und Bewertung der eigenen Zukunftschancen

\begin{tabular}{|c|c|c|c|c|c|c|}
\hline & $N$ & $\begin{array}{l}\text { „aufje- } \\
\text { den Fall“ }\end{array}$ & $\begin{array}{c}\text { "wahr- } \\
\text { schein- } \\
\text { lich" }\end{array}$ & $\begin{array}{c}\text { "nicht si- } \\
\text { cher" }\end{array}$ & $\begin{array}{l}\text { "un- } \\
\text { wabr- } \\
\text { schein- } \\
\text { lich“ }\end{array}$ & $\begin{array}{c}\text { "definitiv } \\
\text { nicht }\end{array}$ \\
\hline $\begin{array}{l}\text { Wird nach Abschluss der Hochschulaus- } \\
\text { bildung als Journalist/in arbeiten* }\end{array}$ & 553 & $22,1 \%$ & $38,2 \%$ & $25,0 \%$ & $8,5 \%$ & $6,4 \%$ \\
\hline $\begin{array}{l}\text { Wird als Journalist/in den Lebensunter- } \\
\text { halt bestreiten können*** }\end{array}$ & 514 & $6,6 \%$ & $42,6 \%$ & $37,5 \%$ & $10,9 \%$ & $2,3 \%$ \\
\hline
\end{tabular}

* Wortlaut der Frage: „Sind Sie der Ansicht, dass Sie nach Abschluss Ihrer Hochschulausbildung als Journalistin bzw. Journalist arbeiten werden?"

** Wortlaut der Frage: „Denken Sie, dass Sie mit einer Tätigkeit als Journalistin bzw. Journalist Ihren Lebensunterhalt bestreiten können?“

Die Studiendauer hat dabei keinen bedeutsamen Einfluss auf die Karriereziele. Anders die Praxiserfahrung: Hier steigt der Anteil der Studierenden, die in den Journalismus wollen, von 31,8 Prozent (keine Praxis) über 56,7 Prozent (max. 6 Monate Praxis) auf 77,8 Prozent. Interessanterweise ist der Prozentsatz derer, die es in den Journalismus zieht, an den Universitäten deutlich und signifikant höher als an Fachhochschulen (74,5 vs. $53,5 \%$ ). Die Unterschiede waren in beiden Fällen hochsignifikant. ${ }^{10}$ Ähnliches gilt für die Einschätzung der Erfolgswahrscheinlichkeit. Waren unter den Studierenden ohne Praxiserfahrung noch 28,9 Prozent der Meinung, sie würden „auf jeden Fall“ oder „wahrscheinlich“ im Journalismus arbeiten, so wächst dieser Anteil über zunächst 54,0 auf schließlich 72,9 Prozent. Auch hier waren sich die Studierenden an den Universitäten

10 Praxiserfahrung: $\chi^{2}(2)=46,16 ; \mathrm{p}<0.001$; Hochschultyp: $\chi^{2}(1)=26,14 ; \mathrm{p}<0.001$ 
$(70,6 \%)$ sicherer als ihre Kommilitonen an den untersuchten Fachhochschulen $(56,3 \%) .{ }^{11}$

Das heißt, eine zunehmende Praxiserfahrung hält junge Menschen keineswegs davon ab, in den Journalismus zu gehen. Im Gegenteil: Die Erfahrungen, die sie im praktischen journalistischen Alltag machen, bestärken die Studierenden darin, den Journalistenberuf zu ergreifen, und geben ihnen überdies auch mehr Sicherheit hinsichtlich der Erreichbarkeit dieses Ziels. Die Praxiserfahrung ist ein entscheidender motivierender Faktor für angehende Journalisten. Dieser Befund steht im krassen Widerspruch zu den Ergebnissen der oben genannten britischen Studie von Hanna und Sanders (2007).

Skeptischer beurteilten die Studierenden allerdings die Aussicht darauf, ihr gesamtes Berufsleben lang im Journalismus tätig zu sein. Von denjenigen, die als Karriereziel den Journalismus angegeben hatten, waren insgesamt nur 54,0 Prozent der Befragten dieser Meinung ( $\mathrm{n}=337$ ). Diese Auffassung bleibt relativ stabil bezüglich Hochschultyp sowie gemessen an Studiendauer und Umfang der Praxiserfahrung. Anhand dieses Ergebnisses lässt sich sagen, dass sich knapp die Hälfte der heutigen Journalismus-Studenten gedanklich bereits darauf eingestellt hat, dass sie an einem bestimmten Zeitpunkt ihres Berufslebens möglicherweise auf andere Tätigkeitsbereiche ausweichen müssen. Für diesen Teil der Befragten bietet der Journalismus also nur eine befristete berufliche Perspektive. Als Gründe führten die Befragten vor allem die allgemein schlechte Berufsperspektive und einen hart umkämpften Arbeitsmarkt an sowie die unsicheren Anstellungsbedingungen, schlechte Bezahlung und mangelhafte Vereinbarkeit von Beruf und Familie (z. B. aufgrund eines hohen Arbeitspensums und wenig planbarer Arbeitszeiten).

Es überrascht daher wenig, dass es unter den Studierenden durchaus einige Verunsicherung darüber gibt, ob sie mit einer Tätigkeit als Journalistin bzw. Journalist in $\mathrm{Zu}-$ kunft tatsächlich ihren Lebensunterhalt bestreiten können. Unter den 337 Befragten, die nach dem Studienabschluss im Journalismus tätig sein wollen, haben 8,9 Prozent geantwortet, dass dem „auf jeden Fall“ so sein werde, und eine Mehrheit von 51,9 Prozent befand dies immer noch für „wahrscheinlich“. Weitere 35,9 Prozent der Studierenden waren sich in dieser Frage allerdings "nicht sicher" und 3,9 Prozent befanden es für „unwahrscheinlich“, dass sie mit Journalismus ihren Lebensunterhalt verdienen können. Während sich diese Auffassung von der Studiendauer dabei unbeeinflusst zeigt, geht der Anteil der Optimisten mit zunehmender Berufserfahrung in der Tendenz zurück (nicht signifikant). An Fachhochschulen ist der Anteil sowohl der Optimisten (52,3 vs. 46,1\%) als auch der Pessimisten höher (16,9 vs. 9,4\%), wohingegen an Universitäten beinahe die Hälfte der befragten Studierenden in dieser Frage unentschieden war (44,5 vs. $30,8 \%)^{12}$

Von den insgesamt 290 Befragten, die als Berufsziel Journalismus und zusätzlich einen konkreten Medienbereich genannt haben, möchte die Mehrheit zum Fernsehen $(51,0 \%)$ gehen, gefolgt von Zeitschriften $(25,5 \%)$ und Zeitungen (14,8 \%). Online-Medien und Nachrichtenagenturen rangieren weit abgeschlagen (6,6 bzw. 2,1 \%). Die überwältigende Mehrheit der Studierenden strebt also immer noch in die klassischen Medienbereiche, was einen interessanten Kontrast zur tatsächlichen Entwicklung der Medienlandschaft bildet. Gemessen am Anteil der Journalisten, die für deutsche Zeitungen tätig sind, scheint dieser Bereich jedoch substanziell an Attraktivität eingebüßt zu haben. Hier drängt sich augenscheinlich ein Zusammenhang mit der aktuellen Zeitungsfinanzierungskrise auf.

11 Praxiserfahrung: $\chi^{2}(2)=39,32 ; \mathrm{p}<0.001$; Hochschultyp: $\chi^{2}(2)=21,24 ; \mathrm{p}<0.001$

$12 \chi^{2}(2)=12,88 ; \mathrm{p}<0.01$. 


\section{Fazit}

Die Krise im Journalismus ist allgegenwärtig und mittlerweile auch im Zentrum gesellschaftlicher und gesellschaftspolitischer Diskurse angekommen. Krisennarrative haben in diesem Kontext Hochkonjunktur in Auslassungen von Berufspraktikern und Journalismusforschern. Betrachtet man Journalismus als Institution, deren soziale Identität und Rolle gesellschaftlich verhandelt wird, stellt sich die Frage, inwiefern diese Krisendebatte Eingang in diesen Aushandlungsprozess findet. Krisennarrative können sich etwa in professionellen und akademischen Diskursen auswirken, an denen Studierende während ihrer Ausbildung teilhaben. Die hochschulgebundene Journalistenausbildung bildet hierbei also gewissermaßen ein „Scharnier“ zwischen professionellen und akademischen Debatten sowie dem breiteren gesellschaftlichen Zeitgespräch. Hier machen viele junge Menschen ihre ersten Erfahrungen mit dem Journalismus und werden allmählich Teil einer diskursiven Praxisgemeinschaft („community of practice“; Gravengaard \& Rimestad 2014), die sie wiederum ihrerseits künftig entscheidend mitprägen werden. Vor dem Hintergrund der aktuell krisenhaften Entwicklung gingen wir daher der Frage nach, wie angehende Journalistinnen und Journalisten die Zukunftschancen des Journalismus bewerten, wo sie die zentralen Herausforderungen und Probleme sehen und was konkret sie in diesen Zeiten in den Journalismus zieht. Darüber hinaus haben wir untersucht, wie viele der Studierenden tatsächlich noch eine Karriere als Journalistin bzw. Journalist anstreben, wie sie in ihre eigene berufliche Zukunft sehen und ob sich Karriereziele und Zukunftseinschätzung im Laufe des Studiums substanziell verändert haben.

Insgesamt konnten wir unter den befragten Studierenden keine ausgeprägte Krisenstimmung ausmachen, was wir als gute Nachricht gelten lassen. Dennoch bewertet eine Mehrheit der Studierenden die Zukunftschancen des Journalismus ambivalent, und der Anteil der Pessimisten überwiegt den der Optimisten. Die größten Herausforderungen für den Journalismus sehen die Befragten erwartungsgemäß vor allem in ökonomischen Faktoren sowie in verschlechterten Arbeitsbedingungen. Es überrascht daher nur wenig, dass angesichts der schwierigen Bedingungen Selbstverwirklichung und Idealismus einen entscheidenden Einfluss auf die Studienwahl von Journalismus-Studierenden hatten und weniger materielle Überlegungen (wie Verdienstmöglichkeiten und Arbeitsplatzsicherheit). Letztere verlieren mit zunehmender Praxiserfahrung sogar noch mehr an Bedeutung. Es liegt nahe, dieses Ergebnis als eine psychologische Reaktion auf die Krise des Journalismus zu interpretieren, gewissermaßen als einen „geschärften Realitätssinn“ oder „pragmatischen Idealismus“ sowie eine folglich verstärkte Hinwendung zu idealistischen Motiven und jenen, die auf Selbstverwirklichung gerichtet sind.

Eine weitere positive Botschaft ist, dass trotz schwieriger Zeiten immer noch eine klare Mehrheit der Studierenden in den Journalismus möchte. Allerdings wird ein nicht unbedeutender Teil der Befragten in andere, wenngleich auch verwandte Berufe ausweichen. Hier entsteht der hochschulgebundenen Journalistenausbildung also durchaus ein gewisser „Streuverlust“. Dieser ist vermutlich auch selbstverschuldet: Denn während der Arbeitsmarkt in seinem bisherigen Kernbereich schrumpft, moniert der Deutsche Journalistenverband eine „quantitative und qualitative Unübersichtlichkeit“ der hochschulgebundenen Ausbildungsangebote (Kaiser 2015: 8). Für Ruß-Mohl (2015: 5) taugt die Journalistenausbildung daher mittlerweile als „Fallstudie organisierter Unverantwortlichkeit“. Wenn Journalismus-Studierende in andere Berufe ausweichen, ist dies möglicherweise eine Selbstkorrektur in einem wild wuchernden Ausbildungsmarkt.

Angesichts der allgegenwärtigen Krisenstimmung im Journalismus könnte man annehmen, dass Praxiserfahrung im Journalismus eher „abschreckend“ auf junge Men- 
schen wirken könnte. Tatsächlich aber halten die Erfahrungen, die Studierende im redaktionellen Alltag machen, die Befragten keineswegs davon ab, das Karriereziel Journalismus zu verfolgen. Im Gegenteil: Praxiserfahrung bestärkt die Studierenden in ihrer Berufswahl und gibt ihnen zudem mehr Sicherheit darüber, dass ihre beruflichen Ziele auch erreichbar sind.

Gleichzeitig macht sich auch hier wieder der geschärfte Realitätssinn bemerkbar: In Zeiten unklarer Berufsaussichten und schwieriger Anstellungsbedingungen bereitet sich knapp die Hälfte der Studierenden im Geiste bereits darauf vor, in einem von beruflicher Mobilität gekennzeichneten Arbeitsmarkt irgendwann auch auf andere Tätigkeitsbereiche auszuweichen. Für diesen Teil der Befragten bietet der Journalismus also nur eine zeitlich befristete berufliche Perspektive.

Die Krisendebatten erreichen die Studierenden also durchaus, viele sind aber willens, sich den erschwerten Bedingungen anzupassen. Vor allem der professionelle Diskurs, zu dem die Studierenden im Zuge erster Praxiserfahrungen Zugang finden, scheint eine positive Vorstellung von Journalismus aufrechtzuhalten und gar zu kultivieren. Dass junge Menschen trotz zahlreicher Nachteile weiterhin in den Journalismus wollen, ist durchaus bemerkenswert. Nach wie vor übt Journalismus als Beruf eine starke Anziehungskraft vor allem auf diejenigen aus, die sich in ihrer Tätigkeit kreativ selbst verwirklichen und - zumindest zum Teil - auch in die Gesellschaft hineinwirken möchten.

\section{Literatur}

Barris, A. (1976). Stop the Presses! The Newspaperman in American Films. New York: Barnes.

BDZV (2012). Die deutschen Zeitungen in Daten und Zablen. http://www.bdzv.de/fileadmin/ bdzv_hauptseite/markttrends_daten/wirtschaftliche_lage/2012/assets/ZahlenDaten_2012.pdf [3.11.2016].

Becker, L. B., Fruit, J. W. \& Caudill, S. (1987). The Training and Hiring of Journalists. Norwood, NJ: Ablex.

Bjørnsen, G., Hovden, J. F. \& Ottosen, R. (2007). Journalists in the Making: Findings from a Longitudinal Study of Norwegian Journalism Students. Journalism Practice, 1(3): 383-403.

Bowers, T. (1974). Student Attitudes toward Journalism as a Major and a Career. Journalism Quarterly, 51(2): 265-270.

Bruns, A. (2005). Gatewatching: Collaborative Online News Production. New York u. a.: Peter Lang.

Burgard, J. P. \& Schröder, M.-M. (2012). Wege in den Traumberuf Journalismus. Deutschlands TopJournalisten verraten ibre Erfolgsgeheimnisse. Münster: Solibro.

Charles, A. \& Stewart, G. (eds.) (2011). The End of Journalism: News in the Twenty-First Centu$r y$. Bern: Peter Lang.

Chilton, P. \& Schäffner, C. (2011). Discourse and Politics. In T. van Dijk (ed.), Discourse Studies: A Multidisciplinary Approach (S. 303-330). London: Sage.

DJV (2009). Arbeitsbedingungen freier Journalisten. Bericht zu einer Umfrage unter freien Journalisten. http://www.djv.de/fileadmin/user_upload/Freiendateien/Freie-Hintergrund/Umfra ge2008.pdf [3.11.2016].

DJV (2014). DJV-Umfrage Freie Journalisten 2014. Berlin: Deutscher Journalisten-Verband.

Donsbach, W., Rentsch, M., Schielicke, A. M. \& Degen, S. (2009). Entzauberung eines Berufs. Was die Deutschen vom Journalismus erwarten und wie sie enttäuscht werden. Konstanz: UVK Medien.

Ehrlich, M. C. (2004). Journalism in the Movies. Chicago: University of Illinois Press.

Esslinger, D. (2009). Journalistenberuf: Journalistik, ein Leerfach. sueddeutsche.de, 16.8.2009, http://www.sueddeutsche.de/karriere/journalistenberuf-journalistik-ein-leerfach-1.166697 [3.11.2016]. 
Europäisches Parlament (2013). EU-Charta: Normensetzung für die Freibeit der Medien in der $E U$. http://www.europarl.europa.eu/sides/getDoc.do?type=TA\&reference=P7-TA-2013-02 03\&language=DE\&ring=A7-2013-0117 [3.11.2016].

Foucault, M. (1973). Archäologie des Wissens. Frankfurt/M.: Suhrkamp.

Fröhlich, R. \& Holtz-Bacha, C. (2009). The German Journalism Education Landscape. In G. Terzis (ed.), European Journalism Education (S. 131-147). London: Routledge.

Gerhardt, R., Kepplinger, H. M. \& Maurer, M. (2005). Klimawandel in den Redaktionen. FAZ, 31.03.2005, S. 40.

Gravengaard, G. \& Rimestad, L. (2014). Socializing Journalist Trainees in the Newsroom: On How to Capture the Intangible Parts of the Process. Nordicom Review, 35: 81-95.

Greck, R. \& Altmeppen, K.-D. (2012). Traumberuf oder Berufstraum(a)? Ausbildungssituation und Berufsrealität von Journalisten. In K.-D. Altmeppen \& R. Greck (Hrsg.), Facetten des Journalismus: Theoretische Analysen und empirische Studien (S. 21-28). Wiesbaden: VS.

Hall, P. A. \& Taylor, R. C. R. (1996). Political Science and the Three New Institutionalisms. Political Studies, 44(5): 936-957.

Hanitzsch, T. \& Vos, T. P. (2016). Journalistic Roles and the Struggle over Institutional Identity: the Discursive Constitution of Journalism. Communication Theory (im Druck).

Hanna, M. \& Sanders, K. (2007). Journalism Education in Britain: Who Are the Students and What Do They Want? Journalism Practice, 1(3): 404-420.

Harnischmacher, M. (2010a). Journalistenausbildung im Umbruch. Zwischen Medienwandel und Hochschulreform. Deutschland und USA im Vergleich. Konstanz: UVK.

Harnischmacher, M. (2010b). Journalistenausbildung im Wandel. Der Einfluss des Bologna-Prozesses auf die Studienangebote an deutschen Hochschulen. Communicatio Socialis, 43(4): 349-367.

Henning, C. \& Henning, W. (2010). Studienführer Journalistik, Kommunikations- und Medienwissenschaften (5. Aufl.). Eibelstadt: Lexika.

Hovden, J. F., Bjørnsen, G., Ottosen, R., Willig, I. \& Zilliacus-Tikkanen, H. (2009). The Nordic Journalists of Tomorrow. An Exploration of First Year Journalism Students in Denmark, Finland, Norway and Sweden. Nordicom Review, 30(1): 149-165.

Howarth, D. (2000). Discourse. Buckingham: Oxford University Press.

IfD Allensbach (2013). Hohes Ansehen für Ärzte und Lehrer-Reputation von Hochschulprofessoren und Rechtsanwälten rückläufig. Allensbacher Berufsprestige-Skala 2013. Allensbach am Bodensee. http://www.ifd-allensbach.de/uploads/tx_reportsndocs/PD_2013_05.pdf [3.11.2016].

Jarren, O. (2012). Medienkrise oder Tageszeitungsfinanzierungskrise? In O. Jarren, M. Künzler \& M. Puppis (Hrsg.), Medienwandel oder Medienkrise? (S. 165-174). Baden-Baden: Nomos.

Kaiser, U. (2015). Ausbildungsgespräche auf Augenhöhe. Aviso, 61, 8-9.

Köcher, R. (2015). Allensbach-Studie: Mehrheit fühlt sich über Flüchtlinge einseitig informiert. FAZ.NET, 16.12.2015, http://www.faz.net/aktuell/politik/fluechtlingskrise/allensbach-um frage-zu-medienberichterstattung-in-fluechtlingskrise-13967959.html [3.11.2016].

Lünenborg, M. (2012). Die Krise des Journalismus? Die Zukunft der Journalistik! Ein Diskussionsbeitrag zur Reflexivität und Praxisrelevanz von Wissenschaft. Publizistik, 57: 445-461.

Marketagent (2010). Einschätzung von Journalisten zur Zukunft ibres Berufes 2010. http://de.sta tista.com/statistik/daten/studie/169725/umfrage/einschaetzung-zur-zukunft-von-journalis ten-in-deutschland/ [3.11.2016].

McChesney, R. \& Pickard, V. (2010). Will the Last Reporter Please Turn out the Lights: The Collapse of Journalism and What Can Be Done To Fix It. New York: New Press.

Meier, K. (2009). Journalismus in Zeiten der Wirtschaftskrise. Neun Thesen zum Strukturwandel der Medien. Journalistik, 1/2009, http://journalistik-journal.lookingintomedia.com/?p=269 [3.11.2016].

Mellado, C., Hanusch, F., Humanes, M. L., Roses, S., Pereira, F., Yez, L., De León, S., Márquez, M., Subervi, F. \& Wyss, V. (2013). The Pre-Socialization of Future Journalists: An Examination of Journalism Students' Professional Views in Seven Countries. Journalism Studies, 14(6): 857-874. 
Neuberger, C. (2002). Journalistikstudium und Arbeitsmarkt. Erfabrungen und Urteile der Eichstätter Journalistik-Absolventen. Eichstätt: KU Eichstätt.

Neuberger, C. (2005). Die Absolventenbefragung als Methode der Lehrevaluation in der Kommunikationswissenschaft. Eine Synopse von Studien aus den Jahren 1995 bis 2004. Publizistik, 50(1): 74-103.

Neuberger, C. (2010). Ein erfolgreicher Start. Auswirkungen der Reform auf die Employability von Absolventen. Aviso, 51: 7-8.

North, D. C. (1991). Institutions. Journal of Economic Perspectives, 5: 97-112.

Nygren, G. \& Stigbrand, K. (2014). The Formation of a Professional Identity. Journalism Studies, 15(6): 841-858.

Nygren, G., Degtereva, E. \& Pavlikova, M. (2010). Tomorrow's Journalists: Trends in the Development of the Journalistic Profession as Seen by Swedish and Russian Students. Nordicom Review, 31(2): 113-133.

Panizza, F. \& Miorelli, R. (2013). Taking Discourse Seriously: Discursive Institutionalism and PostStructuralist Discourse Theory. Political Studies, 61: 301-318.

Patalong, F. (2015). Arbeitsmarkt Journalismus. Wie macht man Karriere in Krisestan? SpiegelOnline, http://www.spiegel.de/karriere/berufsstart/journalist-werden-wie-sieht-es-mit-job-inden-medien-aus-a-1004915.html [3.11.2016].

Röper, H. (2012). Zeitungsmarkt 2012: Konzentration erreicht Höchstwert. Media Perspektiven, 5/2012: 268-285.

Rössler, P., Hautzer, L. \& Lünich, M. (2014). Mediennutzung im Zeitalter von Social Navigation. Ein Mehrebenen-Ansatz zur theoretischen Modellierung von Selektionsprozessen im Internet. In W. Loosen \& M. Dohle (Hrsg.), Journalismus und (sein) Publikum (S. 91-112). Wiesbaden: VS Verlag für Sozialwissenschaften.

Ruß-Mohl, S. (2015). Der überfällige Ruck im Fach. Aviso, 61, 4-5.

Sanders, K., Hanna, M., Berganza, M. R. \& Sánchez Aranda, J. J. (2008). Becoming Journalists: A Comparison of the Professional Attitudes and Values of British and Spanish Journalism Students. European Journal of Communication, 23(2): 133-152.

Schmidt, V. A. (2008). Discursive Institutionalism: The Explanatory Power of Ideas and Discourse. Annual Review of Political Science, 11: 303-326.

Schnedler, T. (2013). San Precario an der Weser. Message, 3/2013: 36-39.

Singer, J. B., Domingo, D., Heinonen, A., Hermida, A., Paulussen, S., Quandt, T., Reich, Z. \& Vujnovic, M. (2011). Participatory Journalism: Guarding Open Gates at Online Newspapers. Oxford: Wiley-Blackwell.

Splichal, S. \& Sparks, C. (1994). Journalists for the 21st Century: Tendencies of Professionalization among First-year Students in 22 Countries. Norwood, NJ: Ablex.

Wagner, R. F. (2009). Lernen und Motivation. In R. F. Wagner, A. Hinz, A. Rausch \& B. Becker (Hrsg.), Modul Pädagogische Psychologie (S. 23-55). Stuttgart: UTB.

Weischenberg, S., Malik, M. \& Scholl, A. (2006). Die Souffleure der Mediengesellschaft: Report über die Journalisten in Deutschland. Konstanz: UVK.

Westerbarkey, J. \& Winkelbrandt, F. (1996). Quo Vadis? Perspektiven von aktiven und ehemaligen Publizistikstudierenden. Münster: Lit.

Zelizer, B. (1993). Journalists as Interpretive Communities. Critical Studies in Mass Communication, 10: 219-237. 
Anhang: Übersicht über die untersuchten Ausbildungseinrichtungen

\begin{tabular}{lllcr}
\hline Hochschule & Standort & Hochschultyp & Studiengang & N \\
\hline BiTS Iserlohn & Iserlohn & Fachhochschule & BA & 29 \\
Hochschule Bremen & Bremen & Fachhochschule & BA & 41 \\
Hochschule Darmstadt & Darmstadt & Fachhochschule & BA & 39 \\
& & & MA & 12 \\
Hochschule Macromedia & Hamburg/Köln & Fachhochschule & BA & 56 \\
Jade Hochschule & Wilhelmshaven & Fachhochschule & BA & 108 \\
Katholische Universität & Eichstätt & Universität & BA & 48 \\
Eichstätt-Ingolstadt & & MA & 14 \\
LMU München & München & Universität & MA & $6 *$ \\
Technische Universität & Dortmund & Universität & BA & 121 \\
Dortmund & & & MA & 10 \\
Universität Hamburg & Hamburg & Universität & BA & 1 \\
& & & MA & 20 \\
Universität Leipzig & Leipzig & Universität & MA & 20 \\
Universität Mainz & Mainz & Universität & MA & 31 \\
\hline
\end{tabular}

* Die LMU-Stichprobe war deutlich kleiner, da die Studie als Lehrprojekt gemeinsam mit Studierenden des MA-Studiengangs Journalismus realisiert wurde.

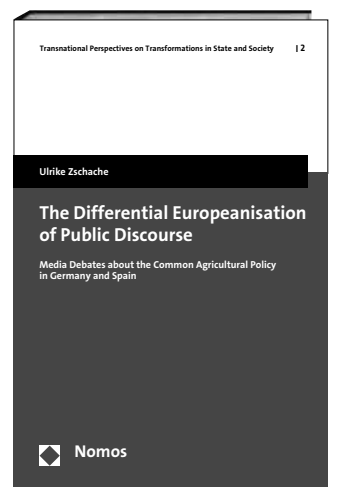

\section{The Differential Europeanisation of Public Discourse}

Media Debates about the Common Agricultural Policy in Germany and Spain

Von Dr. Ulrike Zschache

2016, 442 S., brosch., 84,-€

ISBN 978-3-8487-3443-6

eISBN 978-3-8452-7777-6

(Transnational Perspectives on Transformations in State and Society. Transformation von Staat und Gesellschaft in transnationalen Räumen, Bd. 2) nomos-shop.de/28205

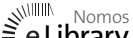 eLibrary

